Pré-Publicações do Departamento de Matemática

Universidade de Coimbra

Preprint Number 10-28

\title{
CONGRUENCES AND IDEALS ON PEIRCE ALGEBRAS: A HETEROGENEOUS/HOMOGENEOUS POINT OF VIEW
}

\author{
SANDRA MARQUES PINTO AND M.TERESA OLIVEIRA-MARTINS
}

\begin{abstract}
For a Peirce algebra $\mathcal{P}$, lattices $\operatorname{Cong} \mathcal{P}$ of all heterogenous Peirce congruences and $I d e \mathcal{P}$ of all heterogenous Peirce ideals are presented. The notions of kernel of a Peirce congruence and the congruence induced by a Peirce ideal are introduced to describe an isomorphism between $C o n g \mathcal{P}$ and $I d e \mathcal{P}$. This isomorphism leads us to conclude that the class of the Peirce algebras is ideal determined. Opposed to Boolean modules case, each part of a Peirce ideal $I=\left(I_{1}, I_{2}\right)$ determines the other one. A similar result is valid to Peirce congruences. A characterization of the simple Peirce algebras is presented coinciding to that given by Brink, Britz and Schmidt in a homogeneous approach.
\end{abstract}

KeYwords: Relation algebras; boolean modules; Peirce algebras; Peirce heterogeneous congruence; Peirce heterogeneous ideal; simple Peirce algebras.

AMS Subject Classification (2010): 03B70, 03G05, 03G15, 06B10, 06E25, 08A68.

\section{Introduction}

Boolean modules were defined and studied by Brink in [1]. A Boolean module is a two-sorted algebra $\mathcal{M}=(\mathcal{B}, \mathcal{R},:)$ of a Boolean algebra $\mathcal{B}$ and a relation algebra $\mathcal{R}$ (Tarski [9] and Chin and Tarski [3]) that are combined by an operator : (the Peircean operator) a map $\mathcal{R} \times \mathcal{B} \rightarrow \mathcal{B}$ taking a relation algebra element and a Boolean algebra element and returning a Boolean algebra element.

A Peirce algebra $\mathcal{P}=\left(\mathcal{B}, \mathcal{R},:,^{c}\right)$ is a Boolean module $(\mathcal{B}, \mathcal{R},:)$ with an additional operator ${ }^{c}$ (the right cylindrification) a map $\mathcal{B} \rightarrow \mathcal{R}$ that creates a relation algebra element from a Boolean algebra element. There is a close relationship between the class of relation algebras and the class of Peirce algebras since every relation algebra gives rise to a Peirce algebra. In [2], Brink, Britz and Schmidt defined a simple Peirce algebra as a Peirce algebra whose underlying Boolean module is simple. There they claim that this definition is equivalent to requiring that the underlying relation algebra is

Received July 29, 2010.

Financial support from the CMUC, Department of Mathematics, University of Coimbra, 3001454 Coimbra, Portugal, is gratefully acknowledged. 
simple. But a homogeneous approach was taken on the characterization of a simple Boolean module. A heterogeneous approach will be taken throughout our work: in the study of the lattices of heterogeneous Peirce congruences and of heterogeneous Peirce ideals and in the classification of the simple Peirce algebras.

\section{Preliminaries}

Peirce algebras are closely related to Boolean modules. We present here the required notions to establish the definition of a Peirce algebra.

Definition 2.1. A relation algebra is an algebra $\mathcal{R}=\left(R, \vee, \wedge,{ }^{\prime}, \mathrm{o}, 1, ;,{ }^{\prime}, e\right)$ satisfying for each $a, b, c \in R$ the following axioms

$\mathrm{R} 1 \quad\left(R, \vee, \wedge,{ }^{\prime}, \mathrm{o}, 1\right)$ is a Boolean algebra

R2 $a ;(b ; c)=(a ; b) ; c$

R3 $a ; e=a=e ; a$

R4 $\quad a^{\llcorner} \backsim=a$

R5 $\quad(a \vee b) ; c=a ; c \vee b ; c$

R6 $\quad(a \vee b)^{\lrcorner}=a^{\triangleleft} \vee b^{\triangleleft}$

R7 $\quad(a ; b)^{\lrcorner}=b \breve{;} a$

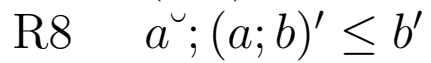

Notation. For $a, b \in R$ we also write $a b$ instead of $a ; b$.

As usual, for every elements $p, q$ on a Boolean algebra $B$ we define $p \oplus q=$ $\left(p \wedge q^{\prime}\right) \vee\left(p^{\prime} \wedge q\right)$. In particular, for every elements $a, b$ on a relation algebra $R$ we define $a \oplus b=\left(a \wedge b^{\prime}\right) \vee\left(a^{\prime} \wedge b\right)$.

The standard class of models of relation algebras is the class of proper relation algebras.

Definition 2.2. A proper relation algebra over a non-empty set $U$ is a set of binary relations on $U$ that contains the identity relation and is closed with respect to union, intersection, complementation, relational composition and converse. If a proper relation algebra consists of all binary relations defined on $U$, then this algebra is called the full relation algebra and is denoted by $\mathcal{R}(U)$. More precisely, $\mathcal{R}(U)$ is the power set algebra over $U^{2}$ endowed with composition (";"), converse ("U") and identity ("Id") operations defined, for $a, b \subseteq U^{2}$, by

$a ; b=\{(s, t):$ exists $u \in U$ such that $(s, u) \in a$ and $(u, t) \in b\}$

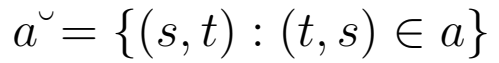

$I d=\{(s, s): s \in U\}$. 
Definition 2.3. An element $a$ of a relation algebra is a right ideal element if and only if $a ; 1=a$.

The arithmetic of relation algebras can be described by the facts assembled on the following theorem.

Theorem 2.4. On any relation algebra $\mathcal{R}$ the following hold for any $a, b, c, d \in$ $R$

$R 9 \quad \breve{e}=e, \quad \breve{o}=o, \quad \breve{1}=1$

$R 10 \quad a \leq b$ if and only if $a^{u} \leq b^{u}$

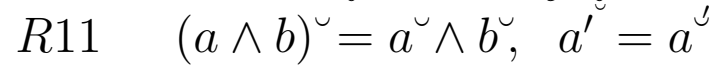

$R 12 \quad a ; o=o=o ; a, 1 ; 1=1$

$R 13 \quad a(b \vee c)=a b \vee a c$

$R 14$ If $a \leq b$ then $c a \leq c b$ and $a c \leq b c$.

$R 15(a b) \wedge c=o$ if and only if $(a \breve{c}) \wedge b=o$ if and only if $(c b) \wedge a=o$

$R 16 \quad(a b) \wedge(c d) \leq a[(a \breve{c}) \wedge(b d y)] d$

$R 17$ If $b$ is a right ideal element then $a \wedge b=(b \wedge e) a$.

$R 18 \quad(a \oplus b)^{\triangleleft}=a^{\triangleleft} \oplus b^{\triangleleft}$

Proof: R9-R16, R17 and R18 are proved in [3], [2] and [6], respectively.

Associated to a relation algebra $\mathcal{R}$ Brink introduced the notion of a Boolean $\mathcal{R}$-module $\mathcal{B}$ as a homogeneous algebra, a Boolean algebra $\mathcal{B}$ where each element of $\mathcal{R}$ define an action on $\mathcal{B}$. The roles of $\mathcal{B}$ and $\mathcal{R}$ as universes of a single two-sorted algebra are taken evenly on the next notion.

Definition 2.5. A Boolean module is a two-sorted algebra $\mathcal{M}=(\mathcal{B}, \mathcal{R},:)$ where $\mathcal{B}$ is a Boolean algebra, $\mathcal{R}$ is a relation algebra and : is a mapping $\mathcal{R} \times \mathcal{B} \longrightarrow \mathcal{B}$ (written $a: p$ ) such that for any $a, b \in R$ and $p, q \in B$ the following assertions are satisfied.

M1 $\quad a:(p \vee q)=a: p \vee a: q$

M2 $\quad(a \vee b): p=a: p \vee b: p$

M3 $\quad a:(b: p)=(a ; b): p$

M4 $e: p=p$

M5 o: $p=0$

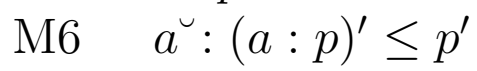

Notation. For $a, b \in R$ and $p \in B$ we also use ap to represent $a: p$.

The standard models of Boolean modules are the proper Boolean modules.

Definition 2.6. A proper Boolean module is a two-sorted algebra of a proper Boolean algebra (a field of sets) and a proper relation algebra together with 
Peirce product defined on sets and relations. For any relation $a$ over some non-empty set $U$ and any subset $p$ of $U$, the Peirce product : of $a$ and $p$ is defined by

$$
a: p=\{s \in U: \text { there exists } t \in p \text { such that }(s, t) \in a\} .
$$

A full Boolean module $\mathcal{M}(U)$ over a non-empty set $U$ is the Boolean module $(\mathcal{B}(U), \mathcal{R}(U),:)$, where $\mathcal{B}(U)$ is the power set algebra over $U, \mathcal{R}(U)$ is the full relation algebra over $U$, and : is the Peirce product defined set-theoretically.

On a Boolean module $\mathcal{M}=(\mathcal{B}, \mathcal{R},:)$ a part of $\mathcal{B}$, the set of all ideal elements, will take a fundamental role later on.

Definition 2.7. Let $\mathcal{M}=(\mathcal{B}, \mathcal{R},:)$ be a Boolean module. An ideal element in $\mathcal{M}$ is a $p \in B$ satisfying $1 p=p$.

Some facts satisfied on Boolean modules deserve mention.

Theorem 2.8. On any Boolean module $\mathcal{M}=(\mathcal{B}, \mathcal{R},:)$ the following hold for any $a, b \in R$ and $p, q \in B$

$$
\begin{aligned}
& M 7 \text { If } p \leq q \text { then } a p \leq a q \text {. } \\
& \text { M8 If } a \leq b \text { then } a p \leq b p \text {. } \\
& M 9 \quad a(p \wedge q) \leq(a p \wedge a q) \\
& M 10 \quad(a \wedge b) p \leq(a p \wedge b p) \\
& \text { M11 ap } \wedge q=0 \text { if and only if } a \breve{q} \wedge p=0 \\
& M 12 \text { If } \sum_{i \in I} p_{i} \text { exists, then so does } \sum_{i \in I} a p_{i} \text {, and } a \sum_{i \in I} p_{i}=\sum_{i \in I} a p_{i} \text {. } \\
& M 13 \quad a 0=0 \\
& M 14 \quad 1: 1=1 \\
& M 15 \quad(a 1)^{\prime} \leq a^{\prime} 1 \\
& \text { M16 ap } \wedge q \leq a(p \wedge a \breve{q}) \\
& M 17 \quad p \leq 1 p \\
& \text { M18 If } p \text { is an ideal element so is } p^{\prime} \text {. } \\
& \text { M19 If } p \text { and } q \text { are ideal elements so is } p \vee q \text {. } \\
& \text { M20 } 1 p \text { is an ideal element. } \\
& M 21 \text { If } p \text { is an ideal element, then } a q \wedge p=a(q \wedge p) \text {. } \\
& \text { M22 If } p \text { is an ideal element, then } a p=a 1 \wedge p \text {. }
\end{aligned}
$$

Proof: Proved in [1]. 


\section{Peirce algebras}

A Peirce algebra $\mathcal{P}=\left(\mathcal{B}, \mathcal{R},:{ }^{c}\right)$ is a Boolean module $(\mathcal{B}, \mathcal{R},:)$ with an additional operator ${ }^{c}$ (the right cylindrification) a map $\mathcal{B} \rightarrow \mathcal{R}$ that creates a relation algebra element from each Boolean algebra element.

Definition 3.1. Let $\mathcal{B}=\left(B, \vee, \wedge,,^{\prime}, 0,1\right)$ be a Boolean algebra and $\mathcal{R}=$ $\left(R, \vee, \wedge,{ }^{\prime}, \mathrm{o}, 1, ;,, e\right)$ be a relation algebra. A Peirce algebra is a two-sorted algebra $\mathcal{P}=\left(\mathcal{B}, \mathcal{R},:{ }^{c}\right)$ where $(\mathcal{B}, \mathcal{R},:)$ is a Boolean module and ${ }^{c}: B \rightarrow R$ is a mapping such that for every $p \in B$ and $a \in R$

$\mathrm{P} 1 \quad p^{c}: 1=p$

$\mathrm{P} 2 \quad(a: 1)^{c}=a ; 1$.

The standard models of Peirce algebras are provided by the class of proper Peirce algebras.

Definition 3.2. A proper Peirce algebra is an algebra $\left(\mathcal{B}, \mathcal{R},:{ }^{c}\right)$ in which $(\mathcal{B}, \mathcal{R},:)$ is a proper Boolean module and ${ }^{c}$ is the cylindrification operation on sets, defined by $p^{c}=p \times V$, for $V$ the universal set of $\mathcal{B}$ and $p$ any subset of $V$.

The full Peirce algebra $\mathcal{P}(U)=\left(\mathcal{B}(U), \mathcal{R}(U),:{ }^{c}\right)$ over some non-empty set $U$ is the full Boolean module $(\mathcal{B}(U), \mathcal{R}(U),:)$ closed with respect to settheoretical cylindrification ${ }^{c}$. Here $p^{c}=p \times U=\{(s, t) \in U \times U: s \in p\}$ for $p$ any subset of $U$.

Example 3.3. The following example can be found on [2]. We can construct a Peirce algebra $\mathcal{P}=\left(\mathcal{B}, \mathcal{R},:,^{c}\right)$ through a relation algebra $\mathcal{R}=$ $\left(R, \vee, \wedge,{ }^{\prime}, \mathrm{o}, 1, ;,,, e\right)$. In fact, if $\mathcal{B}$ is the Boolean algebra of right ideal elements of $\mathcal{R}$, : is ; on $\mathcal{R}$ and ${ }^{c}$ is de map $\mathcal{B} \rightarrow \mathcal{R}$ defined by $p^{c}=p$, then $\mathcal{P}$ is a Peirce algebra.

Later on, a subclass of Peirce algebras will be quite useful.

Definition 3.4. A Peirce algebra $\mathcal{P}=\left(\mathcal{B}, \mathcal{R},:{ }^{c}\right)$ is bijective if and only if, for all $a, b \in R$ we have $a=b$ whenever $a p=b p$ for all $p \in B$.

Theorem 3.5. On any Peirce algebra $\left(\mathcal{B}, \mathcal{R},:{ }^{c}\right)$ the following hold for each $p, q \in B$ and $a, b \in R$

P3 $\quad p^{c}$ is a right ideal element.

P4 $\quad 0^{c}=o, \quad 1^{c}=1$

P5 $\quad(p \vee q)^{c}=p^{c} \vee q^{c}$ 


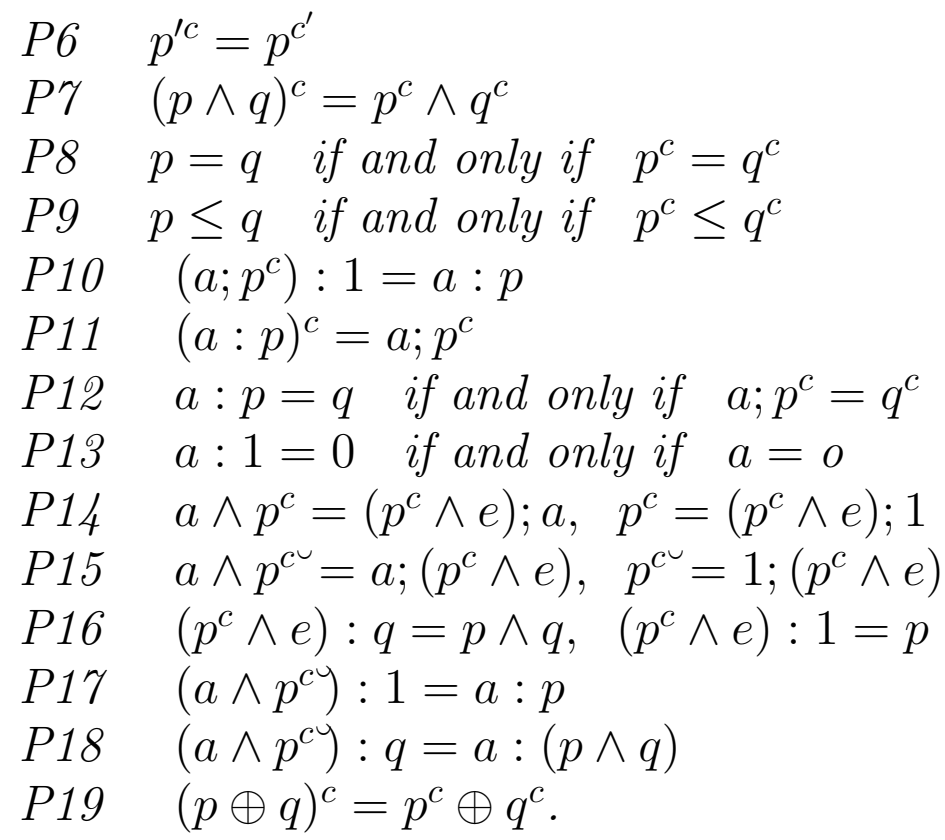

Proof: P3-P18 are proved in [2]. To prove P19 we use P5, P6 and P7. Thus $(p \oplus q)^{c}=\left[\left(p \wedge q^{\prime}\right) \vee\left(p^{\prime} \wedge q\right)\right]^{c}=\left(p \wedge q^{\prime}\right)^{c} \vee\left(p^{\prime} \wedge q\right)^{c}=\left(p^{c} \wedge q^{\prime c}\right) \vee\left(p^{c} \wedge q^{c}\right)=$ $\left(p^{c} \wedge q^{c^{\prime}}\right) \vee\left(p^{c^{\prime}} \wedge q^{c}\right)=p^{c} \oplus q^{c}$.

In [6] we proved that, for $R=\{\mathrm{o}\}$ any Boolean module $\mathcal{M}=(\mathcal{B}, \mathcal{R},:)$ is the degenerate Boolean module. The same proof can be used to validate a similar result for Peirce algebras. Next we present a specific proof to this class of algebras.

Proposition 3.6. In $\mathcal{P}=\left(\mathcal{B}, \mathcal{R},:{ }^{c}\right)$ a Peirce algebra, if $R=\{o\}$, then $\mathcal{P}$ is the degenerate Peirce algebra $\mathcal{P}=\left(\{0\},\{o\},:,^{c}\right)$.

Proof: For any $p \in B$ we have $p^{c} \in R$ and then $p^{c}=0$. Since $0^{c}=0$ then $p^{c}=0^{c}$. By P8 we obtain $p=0$.

\section{The lattice $\operatorname{Cong} \mathcal{P}$}

The lattice of congruences on a given general structure plays a central role both on lattice theory and in the theory of the structure under consideration.

Definition 4.1. Let $\mathcal{P}=\left(\mathcal{B}, \mathcal{R},:{ }^{c}\right)$ be a Peirce algebra. The pair $\theta=\left(\theta_{1}, \theta_{2}\right)$ is a (Peirce) congruence relation on $\mathcal{P}$ if $\theta$ is a modular congruence on the Boolean module reduct of $\mathcal{P}$ and moreover $p^{c} \theta_{2} q^{c}$ whenever $p \theta_{1} q$, i.e., if $\theta_{1}$ is a congruence relation on $\mathcal{B}, \theta_{2}$ is a congruence relation on $\mathcal{R}$, ap $\theta_{1}$ bq whenever $\left(p \theta_{1} q\right.$ and $\left.a \theta_{2} b\right)$ and $p^{c} \theta_{2} q^{c}$ whenever $p \theta_{1} q$. 
Let us denote by $\operatorname{Cong} \mathcal{P}$ the set of all Peirce congruences defined on a Peirce algebra $\mathcal{P}$.

The set $\operatorname{Cong} \mathcal{P}$ is partially ordered by $\left(\theta_{1}, \theta_{2}\right) \leq\left(\gamma_{1}, \gamma_{2}\right)$ if and only if $\theta_{1} \subseteq \gamma_{1}$ and $\theta_{2} \subseteq \gamma_{2}$. Our next aim is to define the lattice structure $\left(\operatorname{Cong} \mathcal{P}, \wedge_{\mathcal{P}}, \vee_{\mathcal{P}}\right)$. Since the intersection $\theta \cap \gamma=\left(\theta_{1} \cap \gamma_{1}, \theta_{2} \cap \gamma_{2}\right)$ of any two Peirce congruences $\theta$ and $\gamma$ defined on $\mathcal{P}$ is, itself, a Peirce congruence on $\mathcal{P}$, let $\theta \wedge_{\mathcal{P}} \gamma=\theta \cap \gamma$. Let us use $\langle\theta\rangle_{\mathcal{A}}$ to represent the congruence relation generated by the binary relation $\theta$ on any (homogeneous or heterogeneous) algebra $\mathcal{A}$, i.e., the intersection of all congruence relations $\theta^{\prime}$ on $\mathcal{A}$ containing $\theta$,

$$
\langle\theta\rangle_{\mathcal{A}}=\cap\left\{\theta^{\prime}: \theta^{\prime} \in C o n g \mathcal{A} \text { and } \theta \subseteq \theta^{\prime}\right\} .
$$

Now we need to define $\theta \vee_{\mathcal{P}} \gamma=\left(\tau_{1}, \tau_{2}\right)$.

Attending to results valid on Boolean modules [6] $\left(\tau_{1}, \tau_{2}\right)$ defined by

$$
\begin{aligned}
\tau_{1} & =\theta_{1} \vee_{\mathcal{B}} \gamma_{1}=\left\langle\theta_{1} \cup \gamma_{1}\right\rangle_{\mathcal{B}} \\
\tau_{2} & =\theta_{2} \vee_{\mathcal{R}} \gamma_{2}=\left\langle\theta_{2} \cup \gamma_{2}\right\rangle_{\mathcal{R}}
\end{aligned}
$$

is a modular congruence on the Boolean module reduct of $\mathcal{P}$ and then $\left(\tau_{1}, \tau_{2}\right)$ will define a Peirce congruence if and only if for every $\theta, \gamma \in C o n g \mathcal{P}$, if $(p, q) \in\left\langle\theta_{1} \cup \gamma_{1}\right\rangle_{\mathcal{B}}$, then $\left(p^{c}, q^{c}\right) \in\left\langle\theta_{2} \cup \gamma_{2}\right\rangle_{\mathcal{R}}$. In fact we have

Proposition 4.2. Let $\mathcal{P}$ be a Peirce algebra. For $\theta=\left(\theta_{1}, \theta_{2}\right), \gamma=\left(\gamma_{1}, \gamma_{2}\right)$ Peirce congruences on a Peirce algebra $\mathcal{P}$, if $(p, q) \in\left\langle\theta_{1} \cup \gamma_{1}\right\rangle_{\mathcal{B}}$, then $\left(p^{c}, q^{c}\right) \in$ $\left\langle\theta_{2} \cup \gamma_{2}\right\rangle_{\mathcal{R}}$.

Proof: Let $(p, q) \in\left\langle\theta_{1} \cup \gamma_{1}\right\rangle_{\mathcal{B}}$. By [4], we know that there exists a natural number $n$, a sequence $p_{1}, p_{2}, p_{3}, \cdots, p_{n}$ of elements in $B$ such that

$$
p \theta_{1} p_{2}, p_{2} \gamma_{1} p_{3}, \cdots, p_{n-1} \gamma_{1} q \text {. }
$$

Since $\theta$ and $\gamma$ are Peirce congruences, then

$$
p^{c} \theta_{2} p_{2}^{c}, p_{2}^{c} \gamma_{2} p_{3}^{c}, \cdots, p_{n-1}^{c} \gamma_{2} q^{c} .
$$

Since $p_{i}^{c} \in R$ for $i=2, \cdots, n-1$ we have $\left(p^{c}, q^{c}\right) \in\left\langle\theta_{2} \cup \gamma_{2}\right\rangle_{\mathcal{R}}$.

The structure $\left(\operatorname{Cong} \mathcal{P}, \wedge_{\mathcal{P}}, \vee_{\mathcal{P}}\right)$ where, for every $\theta, \gamma \in \operatorname{Cong}_{\mathcal{P}}$ the operations are defined by

$$
\begin{aligned}
& \theta \wedge_{\mathcal{P}} \gamma=\theta \cap \gamma=\left(\theta_{1} \cap \gamma_{1}, \theta_{2} \cap \gamma_{2}\right) \\
& \theta \vee_{\mathcal{P}} \gamma=\langle\theta \cup \gamma\rangle_{\mathcal{P}}=\left(\left\langle\theta_{1} \cup \gamma_{1}\right\rangle_{\mathcal{B}},\left\langle\theta_{2} \cup \gamma_{2}\right\rangle_{\mathcal{R}}\right)
\end{aligned}
$$

is a lattice called the congruence lattice $\operatorname{Cong} \mathcal{P}$ of $\mathcal{P}$. 


\section{The lattice $I d e \mathcal{P}$}

Usually ideals and congruences are closely related, in the sense that the zero-class of any congruence is an ideal. Here we present the notion of Peirce ideals that, later on, will enable us to confirm that such a relationship exists for Peirce algebras.

Definition 5.1. A (Peirce) ideal on a Peirce algebra $\mathcal{P}=\left(\mathcal{B}, \mathcal{R},:{ }^{c}\right)$ is a pair $I=\left(I_{1}, I_{2}\right)$ satisfying the following conditions

(1) $I_{1}$ is a Boolean ideal on $\mathcal{B}$;

(2) If $p \in I_{1}$ and $a \in R$ then $p^{c} \in I_{2}$ and $a p \in I_{1}$;

(3) (a) $I_{2}$ is a Boolean ideal on $\mathcal{R}$;

(b) If $a \in I_{2}, c \in R$ then $a c, c a, a \cup \in I_{2}$;

(4) If $a \in I_{2}$ and $p \in B$ then $a p \in I_{1}$.

Such a subset $I_{2}$ of $R$ satisfying condition (3) is called an ideal of $R$.

We note that the pair $I=\left(I_{1}, I_{2}\right)$ is a Peirce ideal on a Peirce algebra $\mathcal{P}=\left(\mathcal{B}, \mathcal{R},:,^{c}\right)$ if it is a modular ideal [6] on the Boolean module reduct of $P$ and if $p^{c} \in I_{2}$ whenever $p \in I_{1}$.

We denote by $I d e \mathcal{P}$ the set of all ideals on a Peirce algebra $\mathcal{P}=\left(\mathcal{B}, \mathcal{R},:{ }^{c}{ }^{c}\right)$. We intend to insert a lattice structure into $I d e \mathcal{P}$. To do so we need to define, for arbitrary Peirce ideals $I$ and $J, I \wedge_{\mathcal{P}} J$ and $I \vee_{\mathcal{P}} J$. It is immediate to put $I \wedge_{\mathcal{P}} J=\left(I_{1} \cap J_{1}, I_{2} \cap J_{2}\right)$.

We denote by $\langle X\rangle_{\mathcal{A}}$ the ideal generated by a subset $X$ of any (homogeneous or heterogeneous) algebra $\mathcal{A}$, i.e., the intersection of all ideals $I$ on $\mathcal{A}$ containing $X$,

$$
\langle X\rangle_{\mathcal{A}}=\cap\{I: I \text { ideal on } \mathcal{A} \text { and } X \subseteq I\} .
$$

In [6] we saw that for $I=\left(I_{1}, I_{2}\right)$ and $J=\left(J_{1}, J_{2}\right)$ elements of $I d e \mathcal{M}$, with $\mathcal{M}=(\mathcal{B}, \mathcal{R},:)$ a Boolean module reduct of $\mathcal{P}=\left(\mathcal{B}, \mathcal{R},:{ }^{c}\right)$ we have

$\left\langle I_{1} \cup J_{1}\right\rangle_{\mathcal{B}}=\left\{p \in B: p \leq p_{1} \vee p_{2}\right.$, for some $\left.p_{i} \in I_{1} \cup J_{1}, i=1,2\right\}$

$\left\langle I_{2} \cup J_{2}\right\rangle_{\mathcal{R}}=\left\{a \in R: a \leq a_{1} \vee a_{2}\right.$, for some $\left.a_{i} \in I_{2} \cup J_{2}, i=1,2\right\}$

Since for $I, J \in I d e \mathcal{P}$ we have $I \vee_{\mathcal{P}} J=\langle I \cup J\rangle_{\mathcal{P}}=\left(\left\langle I_{1} \cup J_{1}\right\rangle_{\mathcal{B}},\left\langle I_{2} \cup J_{2}\right\rangle_{\mathcal{R}}\right)$ on the Boolean module reduct of $\mathcal{P}[6]$ we need to prove that if $p \in\left\langle I_{1} \cup J_{1}\right\rangle_{\mathcal{B}}$ then $p^{c} \in\left\langle I_{2} \cup J_{2}\right\rangle_{\mathcal{R}}$ to infer that this definition is valid on $\mathcal{P}$.

Proposition 5.2. For $\mathcal{P}=\left(\mathcal{B}, \mathcal{R},:{ }^{c}\right)$ a Peirce algebra and $I=\left(I_{1}, I_{2}\right)$ and $J=\left(J_{1}, J_{2}\right)$ Peirce ideals on $\mathcal{P}$ we have $p^{c} \in\left\langle I_{2} \cup J_{2}\right\rangle_{\mathcal{R}}$ whenever $p \in$ $\left\langle I_{1} \cup J_{1}\right\rangle_{\mathcal{B}}$ 
Proof: If $p \in\left\langle I_{1} \cup J_{1}\right\rangle_{\mathcal{B}}$, then there exists $p_{1}, p_{2} \in I_{1} \cup J_{1}$ such that $p \leq p_{1} \vee p_{2}$. Then $p^{c} \leq p_{1}^{c} \vee p_{2}^{c}$ with $p_{1}^{c}, p_{2}^{c} \in I_{2} \cup J_{2}$ (since $I$ and $J$ are Peirce ideals on $\mathcal{P})$.

Therefore, the structure $\mathcal{I} d e \mathcal{P}=\left(\operatorname{Ide} \mathcal{P}, \wedge_{\mathcal{P}}, \vee_{\mathcal{P}}\right)$ with, for every $I=$ $\left(I_{1}, I_{2}\right), J=\left(J_{1}, J_{2}\right) \in I d e \mathcal{P}$, the operations defined by

$$
\begin{aligned}
& I \wedge_{\mathcal{P}} J=I \cap J=\left(I_{1} \cap J_{1}, I_{2} \cap J_{2}\right) \\
& I \vee_{\mathcal{P}} J=\langle I \cup J\rangle_{\mathcal{P}}=\left(\left\langle I_{1} \cup J_{1}\right\rangle_{B},\left\langle I_{2} \cup J_{2}\right\rangle_{R}\right)
\end{aligned}
$$

is a lattice called the lattice of ideals of $\mathcal{P}$.

\section{Peirce congruence versus Peirce ideals}

The purpose of this paragraph is to establish the notions of kernel of a congruence and of Peirce congruence induced by a Peirce ideal. That will enable us to prove the existence of an isomorphism between the lattices Cong $\mathcal{P}$ and $I d e \mathcal{P}$. This isomorphism will lead us to conclude that the class of Peirce algebra is ideal determined [5], i.e., each ideal is the zero-class of a unique Peirce congruence.

Definition 6.1. If $\theta=\left(\theta_{1}, \theta_{2}\right) \in \operatorname{Cong} \mathcal{P}$ where $\mathcal{P}=\left(\mathcal{B}, \mathcal{R},:{ }^{c}\right)$ is a Peirce algebra we say that $\mathcal{I}(\theta)=\mathcal{I}^{\theta}=\left(\mathcal{I}_{1}^{\theta}, \mathcal{I}_{2}^{\theta}\right)$ defined by

$$
\begin{aligned}
& \mathcal{I}_{1}^{\theta}=\left\{p \in B: p \theta_{1} 0\right\}=[0]_{\theta_{1}} \\
& \mathcal{I}_{2}^{\theta}=\left\{a \in R: a \theta_{2} \mathrm{O}\right\}=[\mathrm{o}]_{\theta_{2}}
\end{aligned}
$$

is the kernel of the congruence $\theta$.

Proposition 6.2. The kernel $\mathcal{I}(\theta)$ of a congruence $\theta$ on a Peirce algebra $\mathcal{P}=\left(\mathcal{B}, \mathcal{R},:{ }^{c}\right)$ is an ideal on $\mathcal{P}$.

Proof: Since the kernel of a modular congruence $\theta$ is a modular ideal on the Boolean module reduct of $\mathcal{P}$ [6] we only have to prove that if $p \in \mathcal{I}_{1}^{\theta}$ then $p^{c} \in \mathcal{I}_{2}^{\theta}$. If $p \in \mathcal{I}_{1}^{\theta}$, then $p \theta_{1} 0$. Since $\left(\theta_{1}, \theta_{2}\right)$ is a Peirce congruence $p^{c} \theta_{2} 0^{c}$, i.e., $p^{c} \theta_{2}$ o. So $p^{c} \in \mathcal{I}_{2}^{\theta}$.

Definition 6.3. The kernel of a Peirce homomorphism $h=\left(h_{1}, h_{2}\right)$ : $\mathcal{P} \longrightarrow \mathcal{P}^{\prime}$ between Peirce algebras is the pair $\left(\left\{p \in B: h_{1}(p)=0\right\}\right.$, $\left.\left\{a \in R: h_{2}(a)=\mathrm{o}\right\}\right)$.

Proposition 6.4. The kernel of a Peirce homomorphism $h: \mathcal{P} \longrightarrow \mathcal{P}^{\prime}$ between Peirce algebras is a modular ideal on $\mathcal{P}$.

Proof: Trivial. 
Definition 6.5. If $I=\left(I_{1}, I_{2}\right)$ is a Peirce ideal on a Peirce algebra $\mathcal{P}=$ $\left(\mathcal{B}, \mathcal{R},:{ }^{c}\right)$ we define $\mathcal{C}(I)=\mathcal{C}^{I}=\left(\mathcal{C}_{1}^{I}, \mathcal{C}_{2}^{I}\right)$ by $p \mathcal{C}_{1}^{I} q$ if and only if $p \vee i=q \vee i$ for some $i \in I_{1}$, $a \mathcal{C}_{2}^{I} b$ if and only if $a \vee j=b \vee j$ for some $j \in I_{2}$, for $p, q \in B$ and $a, b \in R$.

Proposition 6.6. If $I=\left(I_{1}, I_{2}\right)$ is a Peirce ideal on a Peirce algebra $\mathcal{P}=$ $\left(\mathcal{B}, \mathcal{R},:{ }^{c}\right)$, then $\mathcal{C}(I)$ is a Peirce congruence on $\mathcal{P}$.

Proof: Since $\mathcal{C}(I)$ is a modular congruence on the Boolean module reduct of $\mathcal{P}$ we only have to prove that if $p \mathcal{C}_{1}^{I} q$ then $p^{c} \mathcal{C}_{2}^{I} q^{c}$. If $p \mathcal{C}_{1}^{I} q$ then there exists $i \in I_{1}$ such that $p \vee i=q \vee i$. So $(p \vee i)^{c}=(q \vee i)^{c}$, i.e., $p^{c} \vee i^{c}=q^{c} \vee i^{c}$. Since $i \in I_{1}$ then $i^{c} \in I_{2}$ and we have $p^{c} \mathcal{C}_{2}^{I} q^{c}$.

Proposition 6.7. If $I=\left(I_{1}, I_{2}\right)$ is an ideal on a Peirce algebra, then $\mathcal{I}(\mathcal{C}(I))=$ $I$.

Proof: Similar to Boolean algebras.

Proposition 6.8. If $\theta=\left(\theta_{1}, \theta_{2}\right)$ is a congruence on a Peirce algebra, then $\mathcal{C}(\mathcal{I}(\theta))=\theta$.

Proof: Similar to Boolean algebras.

Theorem 6.9. The pair of maps $\mathcal{C}: I d e \mathcal{P} \longrightarrow C o n g \mathcal{P}$ (that for each $I \in I d e \mathcal{P}$ assigns the congruence $\mathcal{C}(I)$ ) and $\mathcal{I}: C o n g \mathcal{P} \longrightarrow I d e \mathcal{P}$ (that for each $\theta \in C o n g \mathcal{P}$ assigns the ideal $\mathcal{I}(\theta)$ ) defines an isomorphism between the lattices IdeP $\mathcal{P}$ and $C o n g \mathcal{P}$.

Remark 6.10. Corresponding to any assertion valid for Peirce ideals there exists a valid assertion for Peirce congruence and vice versa.

Remark 6.11. The Peirce algebra class is ideal determined.

\section{Peirce ideal/congruence determined by one of its two parts}

As on Boolean modules [6], on a Peirce algebra $\mathcal{P}=\left(\mathcal{B}, \mathcal{R},:{ }^{c}\right)$, Boolean ideals on $\mathcal{B}$ can exist that are not the Boolean part of any Peirce ideal on $\mathcal{P}$. In fact, let $U=\{p, q\}$ and $\mathcal{P}$ the full Peirce algebra over $U$. The set $I_{1}=\{\emptyset,\{p\}\}$ is a Boolean ideal on $\mathcal{B}(U)$ but, since, for $a \in \mathcal{R}(U)$ given by $a=\{(q, p)\}$, we have $a:\{p\}=\{q\} \notin I_{1}$, so the pair $\left(I_{1}, I_{2}\right)$ is not a Peirce ideal on $\mathcal{P}$, for any subset $I_{2}$ of $R$ (by 2 of Definition 5.1).

This gives rise to the following definitions. 
Definition 7.1. Let $\mathcal{P}=\left(\mathcal{B}, \mathcal{R},:{ }^{c}\right)$ be a Peirce algebra.

(1) A Boolean congruence $\theta_{1}$ on $\mathcal{B}$ is called pro-Peirce congruence on $\mathcal{P}$ whenever there exists a congruence $\theta_{2}$ on $\mathcal{R}$ such that $\left(\theta_{1}, \theta_{2}\right)$ is a Peirce congruence on $\mathcal{P}$.

(2) A Boolean ideal $I_{1}$ on $\mathcal{B}$ is called pro-Peirce ideal on $\mathcal{P}$ if there exists an ideal $I_{2}$ of $\mathcal{R}$ such that $\left(I_{1}, I_{2}\right)$ is a Peirce ideal on $\mathcal{P}$.

Now we give a characterization of pro-Peirce ideals on a Peirce algebra.

Proposition 7.2. On a Peirce algebra $\mathcal{P}=\left(\mathcal{B}, \mathcal{R},:{ }^{c}\right)$ a Boolean ideal $I_{1}$ is a pro-Peirce ideal on $\mathcal{P}$ if and only if the pair $\left(I_{1},\{a \in R:\right.$ ap, a $I_{1}$ for every $\left.p \in B\right\}$ ) is a Peirce ideal on $\mathcal{P}$.

Proof: It is trivial that if the pair $\left(I_{1},\left\{a \in R: a p, a \breve{p} \in I_{1}\right.\right.$ for every $\left.\left.p \in B\right\}\right)$ is a Peirce ideal on $\mathcal{P}$, then the Boolean ideal $I_{1}$ is a pro-Peirce ideal on $\mathcal{P}$.

Now suppose that $I_{1}$ is a pro-Peirce ideal on $\mathcal{P}$ and let $F_{2}=\{a: a p, a \breve{p} \in$ $I_{1}$ for every $\left.p \in B\right\}$. In [6] we proved that $\left(I_{1}, F_{2}\right)$ is a modular ideal on the Boolean module reduct of $\mathcal{P}$ so we only have to prove that if for $p \in I_{1}$ then $p^{c} \in F_{2}$. Since $I_{1}$ is pro-Peirce ideal on $\mathcal{P}$ there exists a ideal $I_{2}$ of $\mathcal{R}$ such that $\left(I_{1}, I_{2}\right)$ is a Peirce ideal on $\mathcal{P}$ and then for $p \in I_{1}$ we have $p^{c} \in I_{2}$ and then $p^{c\llcorner} \in I_{2}$. Since $\left(I_{1}, I_{2}\right)$ is a Peirce ideal then $p^{c} s, p^{c\llcorner} s \in I_{1}$ for every $s \in B$. Therefore $p^{c} \in F_{2}$.

Next example illustrates Proposition 7.2.

Example 7.3. Let $\mathcal{R}$ be the relation algebra with $R=\{\Lambda, a, b, c\}, \Lambda$ the empty relation, $a=\{(p, p)\}, b=\{(q, q)\}$ and $c=\{(p, p),(q, q)\}$ where the operations are defined as in a full relation algebra. Let $\mathcal{P}=\left(\mathcal{B}, \mathcal{R},:,^{c}\right)$ be the Peirce algebra constructed through $\mathcal{R}$ as in Example 3.3. Since every element of $R$ is a right ideal element then $B=\{\Lambda, a, b, c\}$. Let $I_{1}=\{\Lambda, a\}$. Since

$$
\begin{aligned}
& \Lambda \cup \Lambda=\Lambda \Lambda=\Lambda \quad a \backsim \Lambda=a \Lambda=\Lambda \quad b\llcorner\Lambda=b \Lambda=\Lambda \quad c\llcorner\Lambda=c \Lambda=\Lambda
\end{aligned}
$$

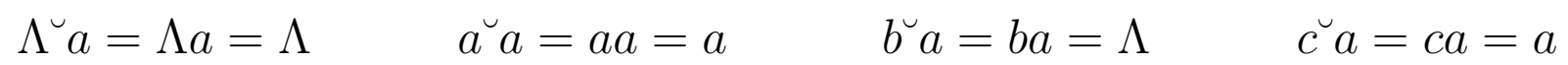

$$
\begin{aligned}
& \Lambda^{\natural} b=\Lambda b=\Lambda \quad a v b=a b=\Lambda \quad b \triangleleft b=b b=b \quad c\llcorner b=c b=b \\
& \Lambda \breve{c} c=\Lambda c=\Lambda \quad \breve{a c}=a c=a \quad \breve{b c}=b c=b \quad c \breve{c}=c c=c
\end{aligned}
$$

then $F_{2}=\left\{d \in R: d p, d p \in I_{1}\right.$ for every $\left.p \in B\right\}=\{\Lambda, a\}$ and so $\left(I_{1}, F_{2}\right)$ is a Peirce ideal on $\mathcal{P}$ and $I_{1}$ is a pro-Peirce ideal. 
Corollary 7.4. On a Peirce algebra $\mathcal{P}=\left(\mathcal{B}, \mathcal{R},:{ }^{c}\right)$ a Boolean congruence $\theta_{1}$ is a pro-Peirce congruence on $\mathcal{P}$ if and only if the pair $\left(\theta_{1},\{(a, b) \in R \times R\right.$ : there exists $j \in R$ such that $a \vee j=b \vee j, j p \theta_{1} 0$ and $j p \theta_{1} 0$ for every $\left.p \in B\right\}$ is a Peirce congruence on $\mathcal{P}$.

Proof: Let $F=\left([0]_{\theta_{1}},\left\{a: a p, a \breve{p} \in[0]_{\theta_{1}}\right.\right.$ for every $\left.\left.p \in B\right\}\right)$. By Proposition 7.2 we know that $[0]_{\theta_{1}}$ is a pro-Peirce ideal on $\mathcal{P}$ if and only if $F$ is a Peirce ideal on $\mathcal{P}$. So it is sufficient to acknowledge that $\mathcal{C}(F)=\left(\theta_{1},\{(a, b) \in R \times R\right.$ : there exists $j \in R$ such that $a \vee j=b \vee j, j p \theta_{1} 0$ and $j p \theta_{1} 0$ for every $p \in$ $B\})$.

In the theory of Boolean modules we recognized [6] the existence, under assumed conditions, of several modular ideals $\left(I_{1}, I_{2}\right)$ with the same Boolean part $I_{1}$. In particular, we were able to construct the smallest and the greatest modular ideals with the same Boolean part. This does not happen for Peirce algebras. In fact, we can establish the following result.

Proposition 7.5. For each pro-Peirce ideal $I_{1}$ on a Peirce algebra $\mathcal{P}=$ $\left(\mathcal{B}, \mathcal{R},:,^{c}\right)$ there exists a unique ideal $I_{2}$ of $\mathcal{R}$ such that $\left(I_{1}, I_{2}\right)$ is a Peirce ideal on $\mathcal{P}$.

Proof: Let $I_{1}$ be a pro-Peirce ideal on $\mathcal{P}$ and suppose that $\left(I_{1}, I_{2}\right)$ and $\left(I_{1}, F_{2}\right)$ are distinct Peirce ideals on $\mathcal{P}$. If $j \in I_{2}$, then $j p \in I_{1}$ for every $p \in B$. In particular, for $p=1$ we have $j 1 \in I_{1}$. Since $\left(I_{1}, F_{2}\right)$ is a Peirce ideal, then $(j 1)^{c} \in F_{2}$. But $(j 1)^{c}=j ; 1^{c}=j ; 1$ and then $j ; 1 \in F_{2}$. Since $e \leq 1$ we have $j ; e \leq j$; 1 , i.e., $j \leq j ; 1$ and so $j \in F_{2}$.

Corollary 7.6. For each pro-Peirce congruence $\theta_{1}$ on a Peirce algebra $\mathcal{P}=$ $\left(\mathcal{B}, \mathcal{R},:{ }^{c}\right)$ there exists a unique congruence $\theta_{2}$ on $\mathcal{R}$ such that $\left(\theta_{1}, \theta_{2}\right)$ is a Peirce congruence on $\mathcal{P}$.

We have already seen that on a Peirce algebra $\mathcal{P}=\left(\mathcal{B}, \mathcal{R},:{ }^{c}\right)$, Boolean ideals on $\mathcal{B}$ can exist that are not the Boolean part of any Peirce ideal on $\mathcal{P}$. We can ask if the same happens for ideals of $\mathcal{R}$, i.e., if there are ideals of $\mathcal{R}$ that are not the relation part of any Peirce ideal on $\mathcal{P}$. Proposition 7.7 states that every ideal of $\mathcal{R}$ is the relation part of a Peirce ideal on $\mathcal{P}$ and gives us the corresponding Peirce ideal construction.

Proposition 7.7. For each ideal $I_{2}$ of $\mathcal{R}$ on a Peirce algebra $\mathcal{P}=\left(\mathcal{B}, \mathcal{R},:,^{c}\right)$ the pair $\left(\left\{p \in B: p^{c} \in I_{2}\right\}, I_{2}\right)$ is a Peirce ideal on $\mathcal{P}$. 
Proof: Let $\mathcal{P}=\left(\mathcal{B}, \mathcal{R},:{ }^{c}\right)$ be a Peirce algebra, $I_{2}$ be an ideal of $\mathcal{R}$ and $I_{1}=\left\{p \in B: p^{c} \in I_{2}\right\}$. Since $0^{c}=o \in I_{2}$ we have $0 \in I_{1}$ and then $I_{1} \neq \emptyset$. Let $p, q \in I_{1}$. then $p^{c}, q^{c} \in I_{2}$ and $(p \vee q)^{c}=p^{c} \vee q^{c} \in I_{2}$. So $p \vee q \in I_{1}$. Let $p \in I_{1}$ and $q \leq p$. Then $q^{c} \leq p^{c}$ and since $p^{c} \in I_{2}$ and $I_{2}$ is an ideal of $R$ we have $q^{c} \in I_{2}$ and then $q \in I_{1}$. Therefore $I_{1}$ is a Boolean ideal on $\mathcal{B}$.

Now we have to prove that if $a \in R$ and $p \in I_{1}$ then $a p \in I_{1}$ and $p^{c} \in I_{2}$. In fact, $(a p)^{c}=a ; p^{c}$ and since $p^{c} \in I_{2}$ and $I_{2}$ is closed by composition by any element of $\mathcal{R}$ we have $a ; p^{c} \in I_{2}$, i.e., $(a p)^{c} \in I_{2}$. So $a p \in I_{1}$. Trivially if $p \in I_{1}$ then $p^{c} \in I_{2}$.

It remains to be proved that if $a \in I_{2}$ and $p \in B$, then $a p \in I_{1}$. In fact, $(a p)^{c}=a ; p^{c}$ and since $a \in I_{2}$ and $I_{2}$ is closed by composition by any element of $\mathcal{R}$ we have $a ; p^{c} \in I_{2}$, i.e., $(a p)^{c} \in I_{2}$ and then $a p \in I_{1}$.

Corollary 7.8. For each congruence $\theta_{2}$ on $\mathcal{R}$ on a Peirce algebra $\mathcal{P}=$ $\left(\mathcal{B}, \mathcal{R},:,^{c}\right)$ the pair $(\{(p, q) \in B \times B$ : there exists $i \in B$ such that $p \vee i=$ $q \vee i$ and $\left.\left.i^{c} \theta_{2} o\right\}, \theta_{2}\right)$ is a Peirce congruence on $\mathcal{P}$.

Proof: For $\theta_{2}$ a congruence on $\mathcal{R}$ we know that $F=\left(\left\{p \in B: p^{c} \in\right.\right.$ $\left.[\mathrm{o}]_{\theta_{2}}\right\},[\mathrm{o}]_{\theta_{2}}$ ) is a Peirce ideal on $\mathcal{P}$ (Proposition 7.7). So it is sufficient to acknowledge that $\mathcal{C}(F)=(\{(p, q) \in B \times B$ : there exists $i \in B$ such that $p \vee i=q \vee i$ and $\left.\left.i^{c} \theta_{2} \mathrm{O}\right\}, \theta_{2}\right)$.

Next example illustrates Proposition 7.7.

Example 7.9. Let $\mathcal{P}=\left(\mathcal{B}, \mathcal{R},:{ }^{c}\right)$ be the Peirce algebra defined in Example 7.3, i.e., $\mathcal{R}$ is the relation algebra with $R=\{\Lambda, a, b, c\}, \Lambda$ the empty relation, $a=\{(p, p)\}, b=\{(q, q)\}$ and $c=\{(p, p),(q, q)\}$ where the operations are defined as in a full relation algebra and $\mathcal{P}$ is the Peirce algebra constructed through $\mathcal{R}$ as in Example 3.3. So $B=\{\Lambda, a, b, c\}$, the Peirce product : is ; on $\mathcal{R}$ and ${ }^{c}$ is de map $\mathcal{B} \rightarrow \mathcal{R}$ defined by $s^{c}=s$ for every $s \in B$. The set $I_{2}=\{\Lambda, a\}$ is a ideal of $\mathcal{R}$ and $\left\{s \in B: s^{c} \in I_{2}\right\}=\left\{s \in B: s \in I_{2}\right\}=\{\Lambda, a\}$. Therefore $\left(\{\Lambda, a\}, I_{2}\right)$ is a Peirce ideal on $\mathcal{P}$ with $I_{2}$ as its relation part.

Proposition 7.5 states that for a pro-Peirce ideal $I_{1}$ on a Peirce algebra $\mathcal{P}=\left(\mathcal{B}, \mathcal{R},:{ }^{c}\right)$ there exists only an ideal $I_{2}$ of $\mathcal{R}$ such that $\left(I_{1}, I_{2}\right)$ is a Peirce ideal on $\mathcal{P}$. Similarly, for any ideal $I_{2}$ of $\mathcal{R}$ we will show that there exists only a Boolean ideal $I_{1}$ on $\mathcal{B}$ such that $\left(I_{1}, I_{2}\right)$ is a Peirce ideal on $\mathcal{P}$.

Proposition 7.10. For each ideal $I_{2}$ of $\mathcal{R}$ on a Peirce algebra $\mathcal{P}=\left(\mathcal{B}, \mathcal{R},:{ }^{c}\right)$ there exists a unique Boolean ideal $I_{1}$ on $\mathcal{B}$ such that $\left(I_{1}, I_{2}\right)$ is a Peirce ideal on $\mathcal{P}$. 
Proof: Proposition 7.7 assures us that, for every ideal $I_{2}$ of $\mathcal{R}$ there exists a Boolean ideal $I_{1}$ on $\mathcal{B}$ such that $\left(I_{1}, I_{2}\right)$ is a Peirce ideal on $\mathcal{P}$. Suppose there is another Boolean ideal $F_{1}$ on $\mathcal{B}$ such that $\left(F_{1}, I_{2}\right)$ is a Peirce ideal on $\mathcal{P}$. If $i \in I_{1}$, then $i^{c} \in I_{2}$. Since $\left(F_{1}, I_{2}\right)$ is a Peirce ideal on $\mathcal{P}$, then $i^{c} p \in F_{1}$ for every $p \in B$. In particular, for $p=1$ we have $i^{c} 1 \in F_{1}$. But by $\mathrm{P} 1$ we have $i^{c} 1=i$, so $i \in F_{1}$.

Corollary 7.11. For each congruence $\theta_{2}$ on $\mathcal{R}$ on a Peirce algebra $\mathcal{P}=$ $\left(\mathcal{B}, \mathcal{R},:,^{c}\right)$ there exists a unique Boolean congruence $\theta_{1}$ on $\mathcal{B}$ such that $\left(\theta_{1}, \theta_{2}\right)$ is a Peirce congruence on $\mathcal{P}$.

\section{Simple Peirce algebras}

A simple Peirce algebra is defined in [2] by Brink, Britz and Schmidt as a Peirce algebra whose underlying Boolean module is simple. And in their characterization of a simple Boolean module a homogeneous approach is taken. Although a heterogeneous point of view was followed on our study, our classification (Proposition 8.3) of a simple Peirce algebra agrees with that reached by them.

Definition 8.1. A Peirce algebra $\mathcal{P}=\left(\mathcal{B}, \mathcal{R},:{ }^{c}\right)$ is simple if and only if Cong $\mathcal{P}=\left\{\left(\Delta_{B}, \Delta_{R}\right),\left(\nabla_{B}, \nabla_{R}\right)\right\}$ (or equivalently, Ide $\left.\mathcal{P}=\{(\{0\},\{\mathrm{o}\}),(B, R)\}\right)$.

Next result, that will be required in the proof of Proposition 8.3, is proved in $[1]$.

Proposition 8.2. On a Boolean module $\mathcal{M}=(\mathcal{B}, \mathcal{R},:)$ a element $p \in B$ is an ideal element if and only if $I_{p}=\{s \in B: s \leq p\}$ is a Boolean ideal on $\mathcal{B}$ satisfying the condition (as $\in I_{1}$ whenever $a \in R$ and $s \in I_{1}$ ).

Proposition 8.3. A Peirce algebra $\mathcal{P}=\left(\mathcal{B}, \mathcal{R},:,^{c}\right)$ is simple if and only if $1: p=1$ for every $p \neq 0$ in $B$.

Proof: Let $\mathcal{P}=\left(\mathcal{B}, \mathcal{R},:{ }^{c}\right)$ be a Peirce algebra where $1: p=1$ for every $p \neq 0$ in $B$ and let be $I_{1} \neq\{0\}$ a pro-Peirce ideal on $\mathcal{P}$. So, there exists a Boolean element $p \neq 0$ such that $p \in I_{1}$. Since $I_{1}$ is pro-Peirce ideal then $a p \in I_{1}$ for every $a \in R$. In particular, for $a=1$ we have $1: p \in I_{1}$. But $1: p=1$, so $1 \in I_{1}$ and so $I_{1}=B$. Trivially $(\{0\},\{0\})$ and $(B, R)$ are Peirce ideals and the only ones with, respectively, Boolean part $\{0\}$ and $B$ (Proposition 7.5). Conversely, suppose that exists $p_{0} \neq 0$ in $B$ such that $1: p_{0} \neq 1$. Let $q_{0}=1: p_{0}$. By M20 we know that $q_{0}$ is a ideal element 
and using Proposition 8.2 we conclude that the set $I_{1}=\left\{s \in B: s \leq q_{0}\right\}$ is a Boolean ideal on $\mathcal{B}$ and $a s \in I_{1}$ whenever $a \in R$ and $s \in I_{1}$. Let $I_{2}=\left\{a \in R: a p \leq q_{0}\right.$ and $a \breve{p} \leq q_{0}$ for every $\left.p \in B\right\}$. We will prove that $\left(I_{1}, I_{2}\right)$ is a Peirce ideal on $\mathcal{P}$. (The use of Proposition 7.2 to prove that $\left(I_{1}, I_{2}\right)$ is a Peirce ideal on $\mathcal{P}$ is not allowed since the assumption of $I_{1}$ being a pro-Peirce ideal on $\mathcal{P}$ is not taken.)

(a) We have to prove that if $s \in I_{1}$, then $s^{c} \in I_{2}$. So we have to prove that if $s \leq q_{0}$, then $\left(s^{c}: p\right) \leq q_{0}$ and $\left(s^{c\llcorner}: p\right) \leq q_{0}$ for every $p \in B$.

We know that $p^{c} \leq 1$ for every $p \in B$ and using R14 we have $q_{0}^{c} ; p^{c} \leq q_{0}^{c} ; 1$. But by $\mathrm{P} 11$ we have $q_{0}^{c} ; p^{c}=\left(q_{0}^{c}: p\right)^{c}$ and since $q_{0}^{c}$ is a right ideal element (P3) we have $q_{0}^{c} ; 1=q_{0}^{c}$. So $\left(q_{0}^{c}: p\right)^{c} \leq q_{0}^{c}$ and by P9 we have $\left(q_{0}^{c}: p\right) \leq q_{0}$ for every $p \in B$. As $s \leq q_{0}$ we have $\left(s^{c}: p\right) \leq\left(q_{0}^{c}: p\right)$ for every $p \in B$, and since $\left(q_{0}^{c}: p\right) \leq q_{0}$ then $\left(s^{c}: p\right) \leq q_{0}$.

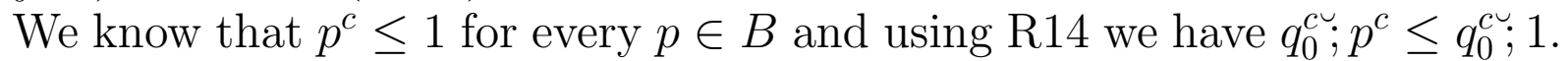

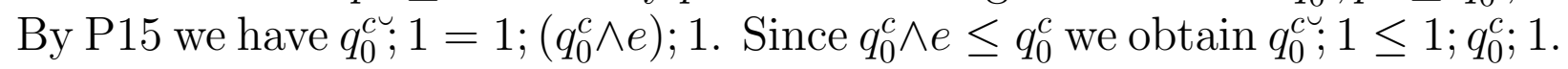
Since $q_{0}$ is a ideal element then $q_{0}=1: q_{0}$ and so $q_{0}^{c}=\left(1: q_{0}\right)^{c}=1 ; q_{0}^{c}$ (by P11). As $q_{0}^{c}$ is a right ideal element (P3) then $1 ; q_{0}^{c}=1 ; q_{0}^{c} ; 1$ and so

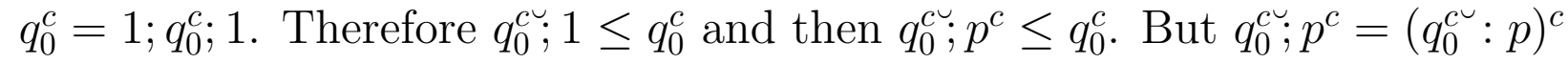
so $\left(q_{0}^{c \sim}: p\right)^{c} \leq q_{0}^{c}$, and by P9 we have $\left(q_{0}^{c \sim}: p\right) \leq q_{0}$. Since $s \leq q_{0}$ we have $\left(s^{c\llcorner}: p\right) \leq\left(q_{0}^{c\lrcorner}: p\right)$ for every $p \in B$ and then $\left(s^{c\llcorner}: p\right) \leq q_{0}$.

(b) We have to prove that the set $I_{2}$ is a Boolean ideal. In fact, o $\in I_{2}$, so $I_{2} \neq \emptyset$.

If $a, b \in I_{2}$, then $a p \leq q_{0}, a \breve{p} \leq q_{0}, b p \leq q_{0}$ and $b \breve{p} \leq q_{0}$ for every $p \in B$. So $(a \vee b) p=a p \vee b p \leq q_{0}$ and $(a \vee b) \breve{p}=a \breve{a} \vee b \breve{p} \leq q_{0}$ and then $(a \vee b) \in I_{2}$.

If $a \in I_{2}$ and $d \in R$ with $d \leq a$, then for every $p \in B$ we have $d p \leq a p \leq q_{0}$ and $d \breve{p} \leq a \breve{p} \leq q_{0}$. So $d \in I_{2}$.

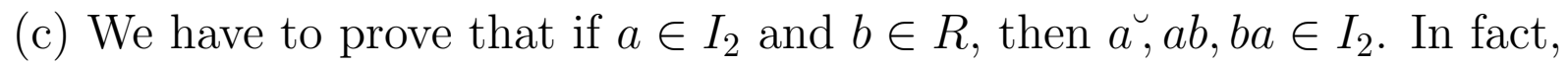
if $a \in I_{2}$, then $a p \leq q_{0}$ and $a \breve{p} \leq q_{0}$ for every $p \in B$. So $a \breve{p}=a p \leq q_{0}$. Therefore $a \breve{v} \in I_{2}$.

Since $a p \leq q_{0}$ for every $p \in B$ then $(a b) p=a(b p) \leq q_{0}$. As $a \breve{p} \leq q_{0}$ then $a \breve{p} \in I_{1}$ and since $d s \in I_{1}$ whenever $\left(d \in R\right.$ and $\left.s \in I_{1}\right)$ we have $(a b) \breve{p}=b(a \breve{p}) \in I_{1}$, i.e., $(a b) \breve{p} \leq q_{0}$. Therefore $a b \in I_{2}$.

Since $a p \leq q_{0}$ for every $p \in B$ then $a p \in I_{1}$, and since $d s \in I_{1}$ whenever $\left(d \in R\right.$ and $\left.s \in I_{1}\right)$ then $(b a) p=b(a p) \in I_{1}$, i.e., $(b a) p \leq q_{0}$. As $a \breve{p} \leq q_{0}$ for every $p \in B$, then $(b a) \breve{p}=a \breve{a}(b \vec{p}) \leq q_{0}$. Therefore $b a \in I_{2}$.

(d) We have to prove that $a p \in I_{1}$ whenever $a \in I_{2}$ and $p \in B$. In fact, if $a \in I_{2}$ then $a p \leq q_{0}$ for every $p \in B$ so $a p \in I_{1}$. 
Therefore $\left(I_{1}, I_{2}\right)$ is a Peirce ideal on $\mathcal{P}$. Since $q_{0} \neq 1$ and $q_{0} \neq 0$ (since by M17 we have $\left.q_{0}=1: p_{0} \geq p_{0} \neq 0\right)$ then $I_{1} \neq B$ and $I_{1} \neq\{0\}$ and so $\mathcal{P}$ is not simple.

Corollary 8.4. Every full Peirce algebra $\mathcal{P}(U)$ over a set $U$ is simple.

Proof: The relation $\nabla_{R} \in \mathcal{R}(U)$ satisfied $\nabla_{R}: p=\nabla_{B}$ for every $p \neq 0$ in $\mathcal{B}(U)$.

Let $\mathcal{P}=\left(\mathcal{B}, \mathcal{R},:{ }^{c}\right)$ be a Peirce algebra where its relation algebra $\mathcal{R}$ contains an element $\exists_{s}$ satisfying $\exists_{s} 0=0$ and $\exists_{s} p=1$ for every boolean element $p \neq 0$. As in Boolean modules, we say that this element of $R$ is the simple quantifier on $\mathcal{P}$.

Remark 8.5. Let $\mathcal{P}=\left(\mathcal{B}, \mathcal{R},:,^{c}\right)$ be a bijective Peirce algebra with the relation algebra $\mathcal{R}$ containing the simple quantifier on $\mathcal{P}$. Then $\exists_{s}=1$ and $1 p=1$ for every $p \neq 0$. (Since for Boolean element $p \neq 0, \exists_{s} p=1$ and $1 p \geq \exists_{s} p=1$ we have $1 p=1$.)

Corollary 8.6. A bijective Peirce algebra $\mathcal{P}=\left(\mathcal{B}, \mathcal{R},:{ }^{c}\right)$ is simple if and only if $\exists_{s} \in R$.

We remark that in [7] we were able to classify the class of simple separable dynamic algebras (following Pratt's definition [8]) as the algebras $\mathcal{D}=$ $\left(\mathcal{B}, \mathcal{R}=\left\{\exists_{s}\right\},\langle\rangle\right)$ with $\mathcal{B}$ arbitrary Boolean algebras. Here, the simple quantifier $\exists_{s}$ played again a central role.

\section{References}

[1] C. Brink. Boolean Modules, Journal of Algebra 71, 291-313(1981).

[2] C. Brink, K Britz, and R. Schmidt. Peirce Algebras, Formal Aspects of Computing 6, 339-358 (1994).

[3] L. H. Chin, and A. Tarski. Distributive and modular laws in the arithmetic of relation algebras, University of California Publications in Mathematics 1, 341-384 (1951).

[4] G. Gratzer. General lattices theory Birkhauser Verlag, 1998.

[5] H.P. Gumm, and A. Ursini. Ideals in universal algebras, Algebra Universalis 19, 45-54 (1984).

[6] S. M. Pinto, and M.T. Oliveira-Martins. Congruences and ideals on Boolean modules: a heterogeneous point of view, (Submitted).

[7] S. M. Pinto, M.T. Oliveira-Martins, and M.C. Pinto. Monadic Dynamic Algebras, Math. Log. Quart. 52, 134-150 (2006).

[8] V.R. Pratt. Dynamic algebras:examples, constructions, applications, In: Technical Report MIT/Laboratory for Comp. Sci/TM-138, pp.1-33 (1979) or Studia Logica 55, 571-605 (1992).

[9] A. Tarski. On the calculus of relations, J.of Symb. Logic 6, 73-89 (1941). 
SANDRA Marques Pinto

Departamento de Matemática, Universidade de Coimbra, 3001-454 Coimbra, PORTUGAL E-mail address: sandra@mat.uc.pt

M.Teresa Oliveira-Martins

Departamento de Matemática, Universidade de Coimbra, 3001-454 Coimbra, PORTUGAL E-mail address: meresa@mat.uc.pt 